\title{
Percent Change From Baseline in Sum of
} Area

National Cancer Institute

\section{Source}

National Cancer Institute. Percent Change From Baseline in Sum of Area. NCI Thesaurus. Code C112369.

The most recently recorded sum of areas minus the baseline sum of areas divided by the baseline sum of areas, multiplied by 100 . 